\title{
Mundo rural, tecnología y producción orgánica
}

\author{
Hernando Zabala Salazar*
}

* Especialista en Derecho Cooperativo. Docente, Fundación Universitaria Luis Amigo, Medellín, Colombia.

Correo electrónico: hernando.zabalasa@amigo.edu.co

Recibido: 15 de junio del 2013 Aprobado: 23 de septiembre del 2013

Cómo citar este artículo: Zabala Salazar, $\mathrm{H}$. (2013). Mundo rural, tecnología y producción orgánica. Cooperativismo \& Desarrollo, 21(103), 81-90.

\section{Resumen}

Gran parte del conflicto político colombiano, traducido en la acción protagónica de grupos armados asentados en territorios de baja presencia estatal, se explica por la existencia de un bajo nivel de desarrollo agrícola en el país y la imposibilidad de hacer realidad una reforma agraria. A lo anterior se agrega el impacto que ha producido la transformación del país en el mundo rural. No es un secreto que la re-estructuración de la economía rural debe ser un proceso de transformación social y regional que termine con el extenso atraso que mantiene al campesino colombiano en las mismas condiciones de finales del siglo XIX. Por ello, es urgente dar respuesta integral a todas estas situaciones y proceder a la formulación de políticas públicas que favorezcan la democratización de la propiedad, la producción con base asociativa, la generación de valores agregados a los productos tradicionales del campo, la producción para la autosuficiencia alimentaria del país, el acceso a los mercados, el mejoramiento del ingreso familiar y la oportunidad para que la población rural se acerque al disfrute de la vida.

Palabras clave: asociatividad, desarrollo local, producción orgánica, ruralidad, tecnología agraria.

\section{The Rural Environment, Technology and Organic Production}

\begin{abstract}
The Colombian conflict —or in other words the leading actions of armed groups based in areas with little state presence - can largely be explained by the low level of agricultural development in the country, and the inability to make agrarian reform a reality. Added to this is the impact that the country's transformation has had on rural areas. It is clear that the restructuring of the rural economy must be a social and regional transformation that puts an end to the enormous delay which has kept Colombia's rural population living in conditions of the late $19^{\text {th }}$ century. It is therefore critical to provide a holistic solution to these situations and formulate public policy that promotes the democratization of property, association-based production, the generation of added value to traditional rural products, production for food self-sufficiency in the country, access to markets, improvement to family income and the chance for the rural population to experience a fuller enjoyment of life.
\end{abstract}

Keywords: associativity, local development, organic production, rural areas, agricultural technology.

\section{Mundo rural, tecnologia e produção orgânica}

\section{Resumo}

Grande parte do conflito político colombiano, traduzido na ação protagônica de grupos armados assentados em territórios de baixa presença estatal, explica-se pela existência de um baixo nível de desenvolvimento agrícola no país e a impossibilidade de tornar realidade uma reforma agrária. Acrescenta-se a isso o impacto que vem produzindo a transformação do país no mundo rural. Não é um segredo que a reestruturação da economia rural deve ser um processo de transformação social e regional que termine com o extenso atraso que mantém o camponês colombiano nas mesmas condições do final do século XIX. Por isso, é urgente dar resposta integral a todas essas situações e proceder à formulação de políticas públicas que favoreçam a democratização da propriedade, a produção com base associativa, a geração de valores agregados aos produtos tradicionais do campo, a produção para a autossuficiência alimentar do país, o acesso aos mercados, o melhoramento do ingresso familiar e a oportunidade para que a população rural se aproxime do desfrutar da vida.

Palavras-chave: associatividade, desenvolvimento local, produção orgânica, ruralidade, tecnologia agrária. 


\section{Una necesaria transformación del mundo rural}

El sistema socioeconómico predominante en la mayoría de los países de la esfera capitalista se sustenta en el principio de la maximización de la inversión, sin medir las consecuencias de los hechos económicos y de los efectos negativos sobre la supervivencia del ecosistema. Ese desbordante afán rentista se ha traducido en un crecimiento acelerado de la producción y el consumo, por encima de los niveles requeridos para satisfacer las necesidades básicas y sin la correspondencia entre el fenómeno productivo humano y el ecosistema.

A la par de la existencia de procesos que generan altos volúmenes de producción de bienes de todo tipo (entre ellos la producción de alimentos que satisface el consumo de sólo una parte de la población mundial), contradictoriamente un inmenso porcentaje de conglomerados humanos carecen de los mínimos alimentarios para su subsistencia. Esta última razón dio pie a que las Naciones Unidas produjera en el 2000 la famosa Declaración sobre los Objetivos de Desarrollo del Milenio, partiendo de señalar que:

no escatimaremos esfuerzos para liberar a nuestros semejantes, hombres, mujeres y niños, de las condiciones abyectas y deshumanizadoras de la pobreza extrema, a la que en la actualidad están sometidos más de 1.000 millones de seres humanos. Estamos empeñados en hacer realidad para todos ellos el derecho al desarrollo y a poner a toda la especie humana al abrigo de la necesidad.

Ese propósito universal de disminuir las condiciones de pobreza - la cual está concentrada en casi todos los países del mundo entre la población rural-, implica no solamente efectuar profundos cambios en la manera como se distribuye la riqueza mediante la producción de políticas públicas que contribuyan a la transformación económica en los órdenes nacionales, propugnando por la disminución de la inequidad, sino también una intervención para otorgar al sector rural un rol adecuado y acorde con los cambios de los últimos tiempos.

La elevación de los ingresos de los trabajadores del campo, producto de su esfuerzo productivo, y la producción de alimentos para mitigar el hambre de millones de pobres, pobladores de territorios urbanos y rurales, son elementos de una misma ecuación. Esto es, el objetivo de disminuir una consecuencia primaria de la pobreza como es la falta de alimentos, aceptado por todos los gobiernos representados en las Naciones Unidas, es una oportunidad gigantesca para realizar transformaciones profundas en la dinámica económica rural.

En el caso de Colombia esto es una verdad de a puño. Por muy variadas circunstancias históricas, el ordenamiento rural colombiano mantiene, en esencia, las mismas condiciones de hace cien años, sin que se haya producido una reforma profunda en sus estructuras. Desafortunadamente la mayoría de los territorios con vocación agraria se sostienen en prácticas básicamente endógenas, sin que se produzca un nexo adecuado con el mundo urbano o con alternativas productivas no relacionadas con la generación de materias primas. Los debates recientes, relacionados con la participación competitiva del país en un mundo de tratados comerciales, indican que es necesario producir, con carácter urgente, un plan de redefinición del rol de la ruralidad.

$\mathrm{Y}$ es que ya para nadie es un secreto que con las transformaciones que se han producido en el mundo durante los últimos treinta años, el medio rural debe entenderse en cumplimiento de otras funciones, muy distintas a las de comienzos del siglo xx. Estas funciones están relacionadas con temas como la conservación del medio ambiente, el equilibrio territorial, la utilización de tecnologías apropiadas, la agroindustria, usos del suelo con funciones diferentes a la producción de materias primas (por ejemplo, para el ecoturismo y la recreación) y la producción de alimentos limpios y orgánicos (Instituto de Ciencia Política, 2011).

Aunque en Colombia este enfoque funcional del campo se da desde comienzos de los años noventa del siglo pasado, son pocos los desarrollos que ha tenido cuando de producir políticas públicas se trata. Sin embargo, es necesario reconocer que en él está la semilla de la disminución de las condiciones de inequidad que ha sufrido el mundo rural colombiano, otorgándole un carácter multifuncional a su rol económico, generándose una nueva visión sobre su desarrollo territorial.

Muchos analistas coinciden en que el modelo económico que se ha configurado en Colombia ha sido discriminatorio respecto del mundo rural, conduciendo a que allí se concentran los mayores índices de pobreza y de falta de acceso a las oportunidades que otorga el desarrollo moderno, y ello es congruente con el hecho de que en estos territorios también se concentren los mayores conflictos y hacen presencia los factores detonantes de la violencia.

La verdad es que esta situación que vive el campo colombiano es absolutamente paradójica, ya que nuestro mundo rural presenta altas potencialidades de 
generación de riqueza. Entre los factores potenciales de Colombia están: a) la gran cantidad de tierras aptas para agricultura en un escenario mundial donde la producción de alimentos cada vez cobra mayor relevancia; b) gran riqueza hídrica, minera y energética con el agua como protagonista en el siglo xxI (por ser un bien público escaso); y c) una megabiodiversidad que genera un elevado potencial en el sector turístico que aún no se desarrolla a plenitud. Si se aprovechan estas oportunidades, ello contribuiría a mejorar la calidad de vida de la población, modernizaría la infraestructura y permitiría el acceso a conocimientos y tecnología que propulsaran la diversificación productiva.

En este proceso transformador de la ruralidad no pueden dejarse de lado muchas circunstancias de adaptación al cambio que deben analizarse con extremo cuidado, tales como el uso de tecnologías apropiadas para lograr el entronque con el mundo industrializado, la aceptación de prácticas agrarias que contribuyan a la sostenibilidad del medio ambiente y la creación de múltiples expresiones de asociatividad productiva que hagan eficiente el proceso económico.

\section{La tecnología como factor económico en el ciclo de producción y como método de sustentabilidad ambiental}

Indudablemente, además de la necesaria producción de políticas públicas (traducidas en normas jurídicas y programas de gobierno), la transformación de la ruralidad colombiana, mediante la adopción de su nuevo enfoque funcional, implica entender el papel que debe cumplir la tecnología en este proceso.

\section{Debates en torno a su significado}

Hoy en día la voz tecnología ha logrado una significación integral respecto de la que se tenía décadas atrás. Ha trascendido el estrecho campo que le otorgara su etimología griega (relacionada directamente con un arte o habilidad) y se viene entendiendo en un sentido más amplio, ofreciéndose una perspectiva unida al amplio espectro del conocimiento. Así pues, hace referencia a la forma de llevar a efecto una determinada acción usando procesos técnicos y métodos sustentados en el conocimiento.

La Enciclopedia Británica anuncia que la tecnología es: "La aplicación del conocimiento científico a los fines prácticos de la vida humana, o como algunas veces se expresa, al cambio y manipulación del entorno humano".

En sentido estricto, la tecnología se entiende como el desarrollo, por medio de la producción del conocimiento en el tiempo, de técnicas sistemáticas para crear y realizar cosas. En el siglo xvir la palabra se utilizaba en relación con las artes aplicadas solamente, pero gradualmente estas mismas artes se convirtieron en el objeto de la designación. A principios del siglo xx, el término abarcó un creciente rango de significados, extendiéndose a la comprensión de procesos e ideas. A mediados del siglo, la tecnología se definió con frases tales como "los medios o actividades mediante los cuales el hombre busca cambiar o manipular su ambiente".

Sin embargo, definiciones tan amplias han sido criticadas ya que dificultan distinguir entre la investigación científica y la actividad tecnológica. Quintanilla (1998) marca la definición de esta manera: "Una realización o aplicación tecnológica es un sistema de acciones humanas, industriales y vinculadas a la ciencia, intencionalmente orientadas a la transformación de objetos concretos para conseguir de forma eficiente resultados valiosos".

$\mathrm{Al}$ ser un sistema, entonces las condiciones iniciales y de contorno influyen en el funcionamiento de la tecnología, siendo influidas por las sociedades que las generan y las impulsan. El ser un sistema de acciones, entonces implica que la filosofía de la tecnología no debe de centrarse en los artefactos o en las máquinas, sino en las acciones que pueden llevarse a cabo gracias a ellas.

En toda acción tecnológica se presupone que el agente dispone de una representación adecuada tanto del objeto sobre el que actúa como de los objetivos que se pretenden con la acción. Precisamente por eso el diseño previo de las acciones es habitual en tecnología, a diferencia de las acciones humanas en general. La existencia de esos diseños, prototipos, representaciones o simulaciones, tienen una gran importancia filosófica ya que las acciones tecnológicas surgen de representaciones más o menos aproximadas de lo que se quiere lograr. Esto requiere de instrumentos, los cuales no son siempre realizables, por lo que la tecnología utiliza los espacios de acciones posibles. También hay que ocuparse de la composición de dichos sistemas, que normalmente se hace por subsistemas para proceder luego a su ensamblaje o integración posterior (Quintanilla, 1988).

Las tecnologías no tratan de describir, explicar o predecir el mundo, a diferencia de las ciencias, sino que tienden a transformarlo, por esta razón la epistemología 
y la filosofía de la ciencia difieren de la filosofía de la tecnología. Las acciones técnicas y tecnológicas, siendo sistémicas, transforman a su vez sistemas, sean estos naturales, sociales, económicos o conceptuales, sean pequeños o grandes. Asimismo, un sistema tecnológico puede transformar profundamente a otro.

Aguilar de la Rosa (2007) explica, con base en una revisión conceptual intensiva, que se puede concluir que el significado de la tecnología se encuentra enmarcado en la actividad productiva y el desarrollo industrial que necesariamente el hombre tiene que planear cuidadosamente para evitar efectos negativos y obtener resultados óptimos de beneficio social.

La definición alude a los objetivos de las acciones tecnológicas que deben ser distinguidos de las acciones de sus agentes. Los artefactos pueden ser diseñados para ciertos propósitos, objetivos o funciones, aunque luego quienes los usan pueden hacerlo con intenciones muy diferentes. En lo que respecta a la eficiencia, esta es relativa, ya que no todas las acciones tecnológicas tienen este valor como preponderante o único. Hay acciones tecnológicas que son altamente ineficientes, y que no por ello dejan de ser tecnológicas (Quintanilla, 1988).

Al hablar de resultados de la tecnología no hay que concentrarse únicamente en los que se observan inmediatamente de las acciones tecnológicas, sino también sus consecuencias y los riesgos derivados. La valoración no solamente se puede hacer desde el punto de vista de los resultados, sino también de las consecuencias imprevistas.

Como lo indica Popper (1974), hay que ser un falseador en la filosofía de la tecnología, una vez que se hayan valorado los resultados favorables, hay que pasar a considerar las posibles consecuencias desfavorables, incluyendo los riesgos que se deriven de los posibles errores en las acciones.

\section{La participación social en los avances tecnológicos}

Respecto de las innovaciones tecnológicas, existen básicamente tres puntos en los cuales debe haber algún involucramiento social: las necesidades sociales, los recursos sociales y un entorno social que simpatice con la idea. Se puede considerar que a la falta de cualquiera de estos factores es poco probable que una innovación tecnológica sea ampliamente adoptada o incluso sea exitosa.

En las sociedades modernas, muchas de las necesidades se generan como consecuencia de la publicidad.
Pero, cualquiera que sea la fuente de la necesidad social, es esencial que suficientes personas sean conscientes de ello, para proveer un mercado para un artefacto o un producto básico de consumo que satisfaga la necesidad.

Los recursos sociales son de la misma manera un prerrequisito indispensable para una innovación exitosa. Entre ellos se pueden mencionar: el capital, los materiales y el personal capacitado. Los recursos de capital involucran la existencia de un excedente de productividad y una organización capaz de dirigir la riqueza disponible en canales mediante los cuales el inventor pueda usarlos. Los recursos de material involucran la disponibilidad de substancias adecuadas que puedan desempeñar cualquier función que una nueva invención requiera de ellas. Los recursos de personal capacitado implican la presencia de técnicos capaces de construir nuevos artefactos y de idear nuevos procesos. Una sociedad tiene que ser bien abastecida con recursos adecuados para poder sostener la innovación tecnológica.

Un ambiente social adecuado implica un ambiente receptivo a nuevas ideas, uno en el cual los grupos sociales dominantes estén preparados a considerar la innovación seriamente. Tal receptividad puede estar limitada a campos específicos o puede tomar la forma de una actitud más generalizada de indagación. No se puede negar que la existencia de grupos sociales importantes dispuestos a animar a los inventores a usar sus ideas ha sido crucial en la historia de la tecnología.

Es imposible negar que exista un elemento de progreso en la tecnología, así como también es claro que la adquisición de técnicas es un asunto acumulativo, en el cual cada generación hereda un conjunto de técnicas sobre las cuales puede seguir construyendo si así lo elige y si las condiciones sociales lo permiten.

A lo largo de mucho tiempo la historia de la tecnología inevitablemente resalta los momentos de innovación que muestra la cualidad de acumulación conforme algunas sociedades avanzan, etapa por etapa de técnicas más primitivas a unas más sofisticadas. Pero aunque este desarrollo ocurre aún, no es intrínseco a la naturaleza de la tecnología que tal proceso de acumulación deberá de ocurrir. El hecho de que muchas sociedades han permanecido estancadas por periodos de tiempo largos, aun en etapas desarrolladas de evolución tecnológica y de que algunas de hecho han perdido terreno y desaprovechado las técnicas acumuladas que habían heredado, pone en evidencia la naturaleza ambigua de la tecnología y la importancia crítica de su relación con otros factores sociales. 
A principios del siglo xvir el filósofo Francis Bacon reconocía innovaciones como la imprenta y el compás magnético, como logros del hombre moderno, además abogaba por la ciencia experimental como un medio de ampliar el dominio del hombre sobre la naturaleza (este es el sentido de su ampliamente conocida utopía social: La Nueva Atlántida). Al enfatizar el papel práctico de la ciencia, Bacon implicó una armonización de la ciencia y la tecnología; él hizo esto explícito al impulsar a los científicos a estudiar el método de los artesanos y a los artesanos a aprender más ciencia. Bacon, junto con Descartes y otros contemporáneos, vieron al hombre como el maestro de la naturaleza y que una convergencia entre las actividades tradicionales de la ciencia y la tecnología era la manera en la cual tal maestría podría lograrse.

En 1660, Un grupo de hombres inspirados en los principios de Bacon formaron la Sociedad Real en Londres representando así un esfuerzo determinado para dirigir la investigación científica hacia fines útiles, primero mejorando la navegación y la cartografía y más tarde para estimular la innovación industrial y la búsqueda de recursos minerales. Cuerpos similares se fueron formando en otros países europeos y para el siglo XIx los científicos se fueron profesionalizando. El papel de personajes como Tomás Edison se hizo significante en profundizar la relación entre ciencia y tecnología; debido a su éxito, llegó a formar el primer laboratorio genuino de investigación industrial.

$\mathrm{Al}$ principio del siglo $\mathrm{xx}$ esto proveyó un modelo que luego aplicaría rigurosamente Henry Ford en su planta de ensamblado de automóviles y que después fue seguido por cualquier proceso de producción en masa moderno. Esto fomentó el desarrollo de ingeniería de sistemas, investigación de operaciones, estudios de simulación, modelación matemática y evaluación tecnológica en procesos industriales.

Esta no es una sola vía de influencia de la ciencia en la tecnología, debido a que la tecnología crea nuevas herramientas y máquinas con las cuales los científicos tienen la posibilidad de lograr una visión cada vez mayor del mundo natural y a su vez pueden generar más tecnología. Sin embargo, actualmente mucho del optimismo del siglo XIX acerca del progreso de la tecnología se ha dispersado y una creciente conciencia del dilema tecnológico que el mundo confronta, hace posible ofrecer una evaluación realista del papel de la tecnología al darle forma a la sociedad al principio del siglo XxI.

Sin embargo, un gran dilema se ofrece para el mundo del porvenir. Por un lado se tiene la gran dependencia de la tecnología en los países avanzados industrialmente, por el otro, la amenaza de que la tecnología destruirá la calidad de vida en la sociedad moderna y aún pondrá en peligro a la sociedad misma. La tecnología confronta a la civilización con la necesidad de tomar una decisión o una serie de decisiones acerca de cómo usar el enorme poder disponible a la sociedad de manera constructiva. La necesidad de controlar el desarrollo de la tecnología y de resolver el dilema, regulando su aplicación a objetivos sociales, hace más necesario definir estos objetivos, mientras los problemas presentados por el rápido crecimiento tecnológico pueda aun resolverse.

Es por esta razón que, muy recientemente, ha surgido el debate sobre el carácter social de la tecnología, naciendo el concepto de innovación social. Mirta Vuotto (2011) explica que propiamente el concepto innovación deriva de la tecnología y de la técnica, diferente al de la innovación social, que está vinculado al vocabulario sociológico. Esta autora latinoamericana concluye que "la innovación social supone una aplicación novedosa de ciertas ideas, incluso si estas no necesariamente son originales, tratándose frecuentemente de una adaptación o de una combinación innovadora de estas ideas o de su aplicación a nuevos dominios".

\section{Tecnología en el mundo agrario}

Las preocupaciones teóricas respecto de los procesos productivos en el mundo agrario surgieron desde la llamada escuela de los fisiócratas, y formó parte importante de las racionalidades de los principales escritos de la escuela liberal. Durante todo el desarrollo de la ciencia económica - hasta nuestros días - el tema ha estado centrado en las relaciones urbano-rurales y se han debatido ampliamente las posibilidades de la industrialización agrícola.

A grandes rasgos, las diferentes escuelas de la economía le otorgaron a la producción rural, por lo menos hasta después de la primera mitad del siglo xx, una función exclusivamente extractiva, restringiendo su papel al aporte de materias primas para el consumo directo, para consumos productivos del agro (por ejemplo, la ganadería), su uso como combustible o para la industria alimenticia y la metalurgia, explicándose los grandes desequilibrios entre los mundos urbano y rural.

Para superar el desequilibrio, por mucho tiempo preponderó la idea de una necesaria industrialización del campo, con base en el principio de localización productiva. Sin embargo, esa perspectiva no tomaba en 
cuenta que las innovaciones tecnológicas no sólo se refieren al escenario de la transformación productiva de materias primas, sino que también se referían a los procesos productivos del mundo rural, para generar una transformación profunda de este mediante reformas integrales en sus estructuras.

Citando al economista John Richard Hicks, el analista chino Pei-Kang Chang (1951) explicaba que los tipos de tecnología pueden clasificarse en tres: los que ahorran trabajo, los que ahorran capital y los neutrales. Sobre esta base indicó Chang que:

todos los tipos de tecnología agrícola pueden clasificarse en tres grupos: 1) el que aumenta sólo la productividad por unidad de superficie, como la introducción de un sistema de rotación, de una nueva raza de ganado, o de nuevas variedades de plantas; 2) el que aumenta sólo la productividad por hora-hombre, como la aplicación de máquinas movidas por energía inanimada [mecanización] y otras formas de equipo agrícola; 3 ) el que aumenta ambas productividades, como el uso de fertilizantes químicos, el control de enfermedades y plagas de las plantas y el ganado, y la adopción de nuevos métodos para evitar la erosión y mantener la fertilidad del suelo.

Estos economistas de la primera mitad del siglo $\mathrm{xx}$ seguían concibiendo el sistema económico sustentado sólo en tres factores productivos: tierra, capital y trabajo. Con sus análisis buscaban la mayor eficiencia y la maximización de dichos factores. En el caso de la producción agraria, el aporte de Chang fue trascendental al enunciar que la productividad no sólo debía restringirse a los factores capital y trabajo, sino que debía extenderse - principalmente - al factor tierra.

Hasta este punto el debate económico se afianzaba en los principios elementales de la economía clásica, y en materia de tecnología predominaba la idea de la introducción de instrumentos físicos (artefactos y máquinas) innovadores del proceso de producción. Pero, pasada la mitad del siglo xx, muy pronto se inauguró la Era del Conocimiento, mediante la cual la propia tecnología se consideró como factor productivo, agregándose a los tres clásicos, y se fue descubriendo un quinto factor: la gestión.

En los tiempos actuales, en consecuencia, hablar de tecnología es hablar de un factor productivo que tiene como función principal la maximización de los demás factores productivos. De ahí que la tecnología ya no podría entenderse restringida, sino identificarse como una fuerza dotadora de productividad y eficiencia en el uso de los factores tierra (hoy entendida como medios materiales), trabajo, capital (entendida en la acepción amplia de medios financieros) y gestión. En este último campo (el de la gestión) los avances tecnológicos están adquiriendo crucial importancia para el desarrollo de las competencias de empresas de todo tipo.

Así pues, el propósito de romper los desequilibrios y resolver la clásica contradicción entre la producción rural y la producción industrial, ya no se resuelve con la simple mecanización o la intensificación de los cultivos o el uso de bienes industrializados aplicados a las técnicas agrícolas, ya que también es de suma importancia realizar aplicaciones tecnológicas al objetivo de la organización misma de la producción (mejorando los métodos de trabajo y la división del trabajo) o atendiendo a la formación de un sexto factor, que por demás es abundante en el mundo rural: la capacidad de actuar comunitariamente y de recoger la memoria colectiva (la cultura productiva de los ancestros).

En estas nuevas dinámicas, relacionadas con el descubrimiento de nuevas funciones para la ruralidad, en las que la producción vegetal es sólo una de ellas, hay que hacer grandes esfuerzos por aumentar los niveles de asociatividad en el campo, suponiendo esta como elemento base de la innovación social. En tal sentido, vale la reflexión de Vuotto (2011) cuando establece que "la innovación social constituye un medio para realizar cambios sistémicos dirigidos a la sociedad, orientándose especialmente a atacar las causas subyacentes de los problemas sociales más que simplemente a atenuar los síntomas".

\section{Producción limpia y producción orgánica: articulaciones y diferencias}

Una buena combinación de ciencia, tecnología e innovación es el tema de la producción orgánica, que se inscribe dentro de un paradigma de desarrollo sustentable, articulado a prácticas ecológicas de producción agropecuaria. Esto es, la producción orgánica puede entenderse como un modo de hacer posible ciertas innovaciones tecnológicas en el quehacer de la economía rural.

La necesidad de una producción agrológica sana, que se ha venido configurando a partir de los problemas del ecosistema y de sus efectos en la salud humana y en el hábitat de la tierra, ha hecho surgir la preocupación por el problema del medio ambiente y ha puesto de relieve la necesidad de sostener la producción, 
cuidando de manera responsable los recursos naturales y garantizando condiciones de vida favorables para las generaciones futuras.

Dentro de un modelo de desarrollo que considera importante la sostenibilidad ambiental, como una de las funciones de la nueva ruralidad, se resalta el auge y posicionamiento que tienen los productos agropecuarios ecológicos u orgánicos, como se puede apreciar con el surgimiento de un mercado dinámico para estos productos, especialmente en los consumidores de los países desarrollados.

\section{Elementos para su conceptualización}

Son diversos los conceptos relacionados con la denominada producción limpia que provienen de la reflexión económica sobre la industria, enmarcados en temas amplios como el de gestión ambiental, vinculados a cualquier cuestionamiento del modelo de desarrollo dominante, en las dimensiones económica, social, política e incluso cultural. La producción limpia en el sector agrícola deriva en otros aspectos que se relacionan con el cuidado de la tierra, del medio ambiente o de la producción alimentaria. En este sentido, se relaciona con la seguridad alimentaria para diversos grupos poblacionales que difieren en sus características según se trate de países desarrollados o en desarrollo. De este modo, también surge la preocupación por los temas de producción agroecológica que son sensibles a la explotación adecuada de los recursos naturales y la producción agrícola con elementos de calidad tradicionales, lo cual incorpora también elementos de connotación cultural y de responsabilidad con el consumo humano, que toca directamente el tema del ambiente y de la salud.

El término producción limpia surge en la década de los años 80 del siglo xx como una respuesta a los crecientes costos derivados de los tratamientos de residuos de las tecnologías de abatimiento o control. Supone un contrasentido al sistema de producción convencional, en el que se genera la mayoría de la contaminación ambiental (externalidades negativas); dicho de otra forma, aparece como término que invita a limpiar lo que ha sido ensuciado. De esta manera, se muestra como un nuevo paradigma y como principio fundamental para el desarrollo de la actividad industrial en un contexto de sostenibilidad económica y ambiental.

La Corporación Autónoma Regional del Centro de Antioquia, plantea que la producción limpia es una opción de gestión ambiental en cada actividad que requiera el uso de los recursos naturales y del medio ambiente, la cual debe incluir la prevención de la contaminación en el origen y la minimización de los residuos, como estrategia que aplica a los procesos, productos y servicios en general.

De su lado, el Instituto para la Educación Ambiental, en Chile, se refiere a la producción limpia a partir de un concepto más amplio que abarca aspectos de la producción, del consumo y del entorno ambiental, que deriva en mayores posibilidades de inserción y de competencia en el mercado, con elementos de responsabilidad en la salud humana. De esta manera, se define como una estrategia de gestión ambiental y empresarial preventiva aplicada a procesos, productos y organización del trabajo; esto es, toca con el concepto de innovación social. Tiene como objetivo la utilización eficiente de las materias primas, la reducción de emisiones y descargas en la fuente misma, la reducción de riesgos para la salud humana y el medio ambiente, con la elevación simultánea de la eficiencia y la rentabilidad de las empresas y, por tanto, su competitividad. Por ello, producir limpio se traduce en sustentabilidad, eficiencia y competitividad de la empresa.

La expresión "producción limpia" tiene un origen, connotación y fin industrial, que debe su aparición a los resultados de las revoluciones industriales del mundo globalizado y al modelo de crecimiento económico. Se constituye como un intento por incorporar consideraciones de tipo ambiental al complejo mundo de los resultados y la eficiencia empresarial.

En el sector agrícola, la producción limpia se refiere a la posibilidad de generar producción por medios que no contaminen el entorno ni perjudiquen la salud humana. Allí se alude al mismo concepto con otras expresiones como agricultura orgánica y agricultura tradicional, entre otras. Sin embargo, dichas alusiones pueden estar en una dimensión distinta a la producción limpia y en contradicción, ya que esta última se inscribe en la misma lógica de desarrollo capitalista sin hacer ruptura con una forma de producción riesgosa para la salud humana y la vida de la tierra. Mientras que la agricultura orgánica corresponde a una práctica milenaria de los agricultores, forma parte de la tradición cultural de la sociedad, vinculada al significado de equilibrio del ciclo natural de la tierra.

En la actualidad, la producción agrícola sustentada en la revolución verde, la relacionada con el incremento de la producción derivada de la aplicación de fertilizantes sintéticos y de productos químicos y nocivos, es cuestionada y se intenta volver a la anterior, 
cuando son muy pocos los que la aplican y queda poca memoria al respecto.

La práctica agrícola más reciente es la producción mixta, híbrida, definida como una forma de cultivo que ejercitan muchos campesinos que combinan actividades tradicionales con elementos de la agricultura moderna. Por su parte, la agricultura tradicional, ancestral o familiar, se realiza en condiciones de marginación y pobreza.

En general, los conceptos relacionados con la agricultura orgánica se articulan a las propuestas que desarrollan los organismos internacionales que intervienen la industria alimentaria en el mundo. De este modo, la Comisión del Codex Alimentarius (FAO) define la agricultura orgánica como "un sistema global de gestión de la producción que fomenta y realza la salud de los agroecosistemas, inclusive la diversidad biológica, los ciclos naturales y la actividad biológica del suelo", lo cual se consigue aplicando, siempre que es posible, métodos agronómicos, biológicos y mecánicos, en contraposición a la utilización de materiales sintéticos, para desempeñar cualquier función específica dentro del sistema.

La Federación Internacional de Movimientos de Agricultura Orgánica (IFOAM), incluye como agricultura orgánica o ecológica a todos los sistemas agrícolas que promuevan la producción sana y segura de alimentos y fibras textiles desde el punto de vista ambiental, social y económico. Estos sistemas parten de la fertilidad del suelo como base para una buena producción, el respeto por las exigencias y capacidades naturales de las plantas, los animales y el paisaje, busca optimizar la calidad de la agricultura y el medio ambiente en todos sus aspectos.

La agricultura orgánica también se entiende como prácticas orientadas hacia una producción sostenible en relación con el medio ambiente, los recursos naturales, la biodiversidad y el bienestar humano. Este tipo de agricultura propugna por la no utilización de insumos externos como los plaguicidas y fertilizantes de síntesis químicas o semillas transgénicas —organismos modificados genéticamente (OMG) - a cambio de realizar las prácticas fitosanitarias y de producción a partir de procesos y controles naturales o biológicos, en busca de obtener producciones limpias, de mayor calidad nutricional, inocuas, que no generen problemas a la salud humana, preservando el entorno o ecosistema.

Las prácticas de agricultura orgánica están soportadas en la sabiduría y el conocimiento de las comunidades rurales ancestrales, relacionadas con los principios de equidad y conservación de todos los elementos de la naturaleza. Las prácticas de producción orgánica surgen en momentos en que la agricultura convencional o comercial está llegando a estados insostenibles en aspectos de productividad y efectos ambientales nocivos a nivel mundial.

Propender por el mantenimiento y la conservación del ecosistema para que la capacidad agrícola alcance desarrollos que puedan ser disfrutables por las generaciones futuras, dio origen al tema de desarrollo sostenible o sustentable, el cual quedó condensado en el Informe Brundtland (elaborado en 1987), definido como el sistema que satisface las necesidades presentes de la humanidad sin comprometer las posibilidades de las generaciones futuras. Este concepto es defendido por la Organización de Naciones Unidas (ONU).

El concepto ha dado origen a varias interpretaciones que fueron incorporándole las distintas dimensiones que se incluyen en los conceptos de bienestar y calidad de vida, tales como la sustentabilidad ecológica, económica, social, política, cultural, entre otros. A partir de la promoción de este paradigma en el discurso del desarrollo, se intentó solucionar las cuestiones más trascendentes e impostergables, tales como la pobreza y la seguridad alimentaria.

En los últimos años el concepto de sostenibilidad se ha centrado en objetivos de desarrollo humano, adquiriendo implicaciones cada vez más precisas, desde el ecodesarrollo y desarrollo sostenible, y ha pasado de ser una preocupación para convertirse en un compromiso de política en la planeación y puesta en marcha de modelos productivos de desarrollo.

Según el Informe Brundtland (pues todo indica que fue en este en el que por primera vez se hizo mención al concepto), el desarrollo sustentable es aquel que satisface las necesidades presentes de la humanidad, sin comprometer en ello las posibilidades de las generaciones futuras o las por venir. No sobra advertir que tal concepto cuenta entre sus más importantes promotores a la Organización de Naciones Unidas (ONU).

El concepto desarrollo sustentable, instalado a partir del Informe Brundtland, realizado y presentado en 1987 por la Comisión Mundial sobre Ambiente y Desarrollo, ha dado origen, a lo largo de los años, a varias interpretaciones, de mayor o menor amplitud, que han ido incorporándose a las distintas dimensiones y concepciones que componen el bienestar de las personas, tales como la sustentabilidad o sostenibilidad ecológica, económica, social, política, cultural, etcétera.

Es así que, a partir de la instalación de este paradigma en los discursos de más variada índole, se 
intenta solucionar las cuestiones más trascendentes e impostergables que aquejan al mundo, como la pobreza (con todo lo que ello significa en materia de salud, educación, seguridad y justicia) y el deterioro ambiental. Tan loables objetivos han generado que desde variados ámbitos y sectores se proclame al desarrollo sustentable como la única salida para que la humanidad y el planeta no sigan precipitándose hacia situaciones de degradación irreversibles.

\section{Tecnología, producción limpia y producción orgánica en el proceso de transformación de la ruralidad colombiana}

Según Estefanía Blount (1998), el modelo de producción vigente en las economías del mundo capitalista es insostenible en sus esquemas de producción y de consumo, debido a que se apoya en formas agresivas de transformación de los recursos naturales que pretenden principalmente la obtención de rentabilidad, sin importar el costo social y las consecuencias negativas sobre el ambiente ni la creciente incapacidad de la naturaleza existente para absorber todos los elementos contaminantes.

La sociedad actual está constantemente atravesada por una cultura consumista depredadora, desconocedora de los procesos de generación de los recursos que intervienen en la producción y la forma en que se desechan los residuos cuando se consideran inútiles.

La forma de producción coherente que intente integrar los procesos industriales y agrícolas dentro de los ciclos de la naturaleza, puede evitar el derroche de los recursos naturales, particularmente de los no renovables, reducir la generación de residuos, vertidos líquidos y emisiones, y preservar la biodiversidad, que se deriva de manera coherente en el cuidado de la salud humana. Estas características se explicitan en las exigencias del mercado internacional para admitir el comercio de los productos (Normas Iso 14.000, calidad, sello verde, etcétera).

Las pequeñas y medianas empresas enfrentan exigencias medioambientales tanto al ser fiscalizadas como al ser evaluada la calidad de sus productos y procesos, o al proteger y asegurar la salud de los trabajadores, para competir por la preferencia de los consumidores $y$, especialmente, al momento de exportar.

Muchos de los problemas relacionados con el medio ambiente y con la sostenibilidad son superables mediante la aplicación de innovaciones con sentido social como la producción orgánica, que es la aplicación de un conjunto de medidas estratégicas orientadas a lograr una mayor eficiencia en el uso de materias primas e insumos para la producción, lo cual redundará en diversos beneficios para el consumidor final, los productores y el exportador, entre otros.

Es por esto que en el esfuerzo transformador de la ruralidad colombiana se proponen políticas públicas que manifiestan la preocupación de las administraciones públicas por generar dinámicas productivas que incorporen nuevos procesos productivos que aprovechen de manera inteligente y sostenible los recursos, para el logro de una calidad de vida digna, sin una visión asistencialista ni reducida a la agricultura (agricultura alimentaria y agroindustria), sino ampliada al aprovechamiento de los recursos naturales, la sostenibilidad del medio ambiente y los servicios ambientales, y la conservación y utilización del agua, entre otros.

Dentro de este contexto, se ubican en Antioquia importantes áreas que tienen una gran riqueza en recursos naturales como agua, bosque, fauna y paisaje. Este departamento está constituido por nueve subregiones; estas son: Valle de Aburrá, Bajo Cauca, Norte, Nordeste, Occidente, Oriente, Urabá, Magdalena Medio y el Suroeste. Esta gran región, en su contexto geopolítico, muestra un rol de gran importancia a nivel nacional, pues cuenta con tres grandes cuencas hidrográficas: la del río Magdalena - que tiene conectividad hacia la cuenca del Caribe, el centro oriente del país y con Venezuela-, la cuenca del Cauca, con un eje urbano que regula la conectividad entre el sur, el centro y el norte del occidente colombiano, y la cuenca del Atrato Norte, que ilustra el corredor biogeográfico del Pacífico y el noroccidente del país. El capital natural y físico que tiene Antioquia la potencia para ser una región de gran desarrollo económico.

De su lado, la subregión del suroeste antiqueño está conformada por cuatro zonas geográficas (o microrregiones): Sinifaná, San Juan, Penderisco y Cartama, las cuales reúnen 23 municipios. De estas zonas, las dos últimas presentan importantes ventajas comparativas dentro del nuevo enfoque funcional de la ruralidad, relacionadas con la producción agropecuaria y las reservas forestales.

Las subregiones antioqueñas requieren la atención de los sectores público y privado para orientar su desarrollo, dadas las potencialidades; pero también las precarias condiciones de vida de una gran mayoría 
de sus habitantes exige un compromiso urgente con la transformación de la estructura rural. Para nadie es un secreto que - excluyendo los 10 municipios que conforman el valle del Aburrá- más del $80 \%$ de los municipios de los restantes (115 en total) registran niveles de pobreza por encima del $80 \%$, lo que a todas luces indica que el modelo vigente, excesivamente concentrador, no sólo ha sido inequitativo sino también poco efectivo en la oferta de soluciones adecuadas que contribuyan a resolver la falta de crecimiento económico y de bienestar de la población rural antioqueña (Plan de Desarrollo de Antioquia 2008-2011).

Mientras el valle de Aburrá genera cerca del 70\% del producto interno bruto (PIB) del departamento, y concentra aproximadamente el $60 \%$ de la población, en tan sólo el 1,8\% del territorio, el suroeste antioqueño concentra el $6 \%$ de la población total y contribuye con el 5,6\% del producto interno bruto departamental; su mayor aporte por rama de actividad económica lo constituye el sector agropecuario, con un $11,56 \%$, representado en una variada producción de café, plátano, yuca, maíz, frutales, hortalizas, fríjol y caña panelera; y en la actividad minera con productos como el oro y el carbón.

El departamento de Antioquia requiere definir un modelo de desarrollo que permita aprovechar el potencial de las subregiones, sustentadas en su posición geoestratégica, el potencial hídrico (cuencas de los ríos Atrato, San Juan y Cauca), la riqueza de zonas de vida, clima y paisajístico, la participación de líderes comunitarios en los procesos de desarrollo, la disposición a la diversificación productiva, el potencial forestal y la presencia de organizaciones crediticias y entidades financieras de economía solidaria, entre otros.

\section{Referencias}

Aguilar de la Rosa, G. (2007). Reflexiones en torno al transcurso de la ciencia y la tecnología en el desarrollo de la humanidad. Recuperado de http://www.publicaciones.ujat.mx/publicaciones/perfiles/ene_abril2006/ Pag.21a39.pdf

Blount Martín, E. (1998). Cerrar los ciclos de producción: un reto clave. Abaco: Revista de Cultura y Ciencias Sociales, 17-18, 51-58. Recuperado de http://dialnet.unirioja.es/ servlet/articulo?codigo $=113160$

Chang, P. K. (1951). Agricultura e industrialización. México: Fondo de Cultura Económica.

Instituto de Ciencia Política (2011). Mesa de expertos: desarrollo rural. ICP, Observatorio Legislativo, Boletín No. 190, Bogotá.

Organización de las Naciones Unidas (onu) (2000). Declaración del Milenio. New York: ONU.

Popper, K. (1974). Conocimiento objetivo. Madrid: Tecnos.

Quintanilla, M. (1998). Técnica y cultura. Tecnos, XVII/3. Recuperado de http://www.oei.es/salactsi/teorema03.htm

Vuotto, M. (2011). La contribución de la investigación a las innovaciones: el caso de las organizaciones de economía solidaria. Innovación y economía social y solidaria. Barranquilla: Ibarra Garrido. 